\title{
Liquid biopsy of extracellular vesicle biomarkers for prostate cancer personalized treatment decision
}

\author{
Meng Han ${ }^{1,2}$, Bairen Pang ${ }^{3,4}$, Cheng Zhou ${ }^{1,2}$, Xin Li ${ }^{1,2}$, Qi Wang ${ }^{3,4}$, Junhui Jiang ${ }^{1,2}$, Yong Li $i^{3,4}$ \\ ${ }^{1}$ Translational Research Laboratory for Urology, the Key Laboratory of Ningbo City, Ningbo First Hospital, The Affiliated Hospital \\ of Ningbo University, Ningbo 315600, Zhejiang, China. \\ ${ }^{2}$ Ningbo Clinical Research Center for Urological Disease, Ningbo 315600, Zhejiang, China. \\ ${ }^{3}$ St George and Sutherland Clinical School, Faculty of Medicine, UNSW Sydney, Kensington, NSW 2052, Australia. \\ ${ }^{4}$ Cancer Care Centre, St. George Hospital, Kogarah, NSW 2217, Australia.
}

\begin{abstract}
Correspondence to: Prof. Yong Li, Cancer Care Centre, St. George Hospital, Level 2, Research and Education Centre, 4-10 South St, Kogarah, NSW 2217, Australia. E-mail: y.li@unsw.edu.au; Dr. Junhui Jiang, Department of Urology, Ningbo First Hospital, The Affiliated Hospital of Ningbo University, Liuting Street 59, Haishu District, Ningbo 315600, Zhejiang, China.

E-mail: Jiangjh200509@126.com
\end{abstract}

\begin{abstract}
How to cite this article: Han M, Pang B, Zhou C, Li X, Wang Q, Jiang J, Li Y. Liquid biopsy of extracellular vesicle biomarkers for prostate cancer personalized treatment decision. Extracell Vesicles Circ Nucleic Acids 2022;3:3-9.
\end{abstract}

https://dx.doi.org/10.20517/evcna.2021.20

Received: 25 Nov 2021 First Decision: 10 Dec 2021 Revised: 20 Dec 2021 Accepted: 4 Jan 2022 Published: 13 Jan 2022

Academic Editors: Yoke Peng Loh, Michael Graner Copy Editor: Yue-Yue Zhang Production Editor: Yue-Yue Zhang

\begin{abstract}
Liquid biopsy of tumor-derived extracellular vesicles (EVs) has great potential as a biomarker source for prostate cancer ( $\mathrm{CaP}$ ) early diagnosis and predicting the stages of cancer. The contents of EVs play an important role in intercellular communication and have specific expression in blood and urine samples from CaP patients. Powered by high-throughput, next-generation sequencing and proteomic technologies, novel EV biomarkers are easily detected in a non-invasive manner in different stages of $\mathrm{CaP}$ patients. These identified potential biomarkers can be further validated with a large sample size, machine learning model, and other different methods to improve the sensitivity and specificity of CaP diagnosis. The EV-based liquid biopsy is a novel and less-invasive alternative to surgical biopsies which would enable clinicians to potentially discover a whole picture of tumor through a simple blood or urine sample. In summary, this approach holds promise for developing personalized medicine to guide treatment decisions precisely for CaP patients.
\end{abstract}

Keywords: Prostate cancer, liquid biopsy, extracellular vesicle, biomarker, early diagnosis, risk prediction 
Prostate cancer $(\mathrm{CaP})$ is one of the most common malignancies in male in Western countries. With economic development and lifestyle changes, the incidence and mortality rate of $\mathrm{CaP}$ among Asian males have been rising rapidly ${ }^{[1,2]}$. In China, $\mathrm{CaP}$ is the leading male genitourinary malignant tumor, and its incidence is now higher than bladder cancer ${ }^{[3]}$. In 2020, CaP caused more than 50,000 deaths in China, which were nearly twice as high as in the United States ${ }^{[4]}$. The survival of CaP patients is highly dependent on tumor stage and risk classification. It was reported that low-risk and localized CaP achieved a favorable prognosis by long-term close monitoring, while metastatic patients had a median survival of 30 months $\mathrm{s}^{[5]}$. The treatment options for different risk classifications are also unique. The principal treatment for clinically low-risk CaP is radical treatment, such as prostatectomy and radiotherapy. In addition to radical treatment, comprehensive treatment such as radiotherapy, chemotherapy, and endocrine therapy is required for patients with high-risk $\mathrm{CaP}$. Therefore, $\mathrm{CaP}$ early diagnosis and grading are crucial to initiate proper treatment, improve patients' outcomes, and prolong survival.

Currently, prostate-specific antigen (PSA) is the most used diagnostic biomarker for CaP diagnosis in the clinic. The widespread use of PSA-testing has increased the diagnostic rate of CaP, but it is also accompanied by a high false-positive rate of $\mathrm{CaP}$ and overtreatment of indolent tumors due to the poor accuracy ${ }^{[6]}$. PSA cannot differentiate among benign prostate changes, indolent cancers (unlikely to cause significant symptoms), and early versus advanced stages of CaP. It often leads to $20 \%$ to $42 \%$ over-diagnosis and over-treatment, which may cause a patient more harmful than good ${ }^{[7]}$. The Prostate Cancer Prevention Trial showed that $14.9 \%$ of prostate tumors in men with PSA levels lower than $4.0 \mathrm{ng} / \mathrm{mL}$ had Gleason scores of seven or higher ${ }^{[8]}$.

Also, due to the limitations of its low specific and low sensitive character, early detection and real-time monitoring of tumor progression cannot be achieved ${ }^{[9]}$. Needle biopsy is the gold standard for CaP diagnosis. However, tumor biopsy has several limitations in clinical application, including the pain associated with the invasiveness of the procedure, the significant risk of hemorrhage and urinary retention, and the risk of false-negative results due to tumor heterogeneity. Magnetic resonance imaging (MRI) has somewhat improved selection for biopsy but the utility is still limited by occasional false negatives in 5\%$15 \%$ of Prostate Imaging-Reporting And Data System (PIRADS) and false positives in 40\%-60\% of PIRADS scores 3-5 (equivocal or positive MRIs) ${ }^{[10]}$. There is thus an unmet need to develop tools to noninvasively detect $\mathrm{CaP}$ at an early stage with high sensitivity and specificity and to improve individualized treatment.

Liquid biopsy refers to the technology that makes full use of body fluids minimally or non-invasively obtained, such as blood, urine, or saliva, and it is receiving great attention as a novel diagnostic tool to access response to clinically and biologically relevant information ${ }^{[11]}$. EV-based liquid biopsy can be integrated to maximize insights into tumor status especially in view of dissecting tumor heterogeneity. Liquid biopsy components include circulating tumor cell, circulating tumor DNA, and/or extracellular vesicles $(\mathrm{EVs})^{[12,13]}$. Compared with other types of liquid biopsy, the use of EVs such as exosomes may offer unique advantages. Firstly, exosomes are highly abundant in most biological fluids, such as blood plasma, where one can detect $10^{8-13}$ exosome particles $/ \mathrm{mL}^{[14]}$. Secondly, tumor-derived exosomes have specific biomarkers distinguished from exosomes from normal tissues, which can be used for cancer diagnosis and prognosis ${ }^{[15]}$. Furthermore, exosomes have strong stability, which means they can be stored at $-80{ }^{\circ} \mathrm{C}$ for several months or even years ${ }^{[16]}$. Thus, exosomes are emerging as a newly attractive biomarker of liquid biopsy for non-invasive cancer diagnosis. EVs are potent and clinically valuable tools for CaP early diagnosis and prognosis as they are highly representative of their cell of origin ${ }^{[17]}$. 
It was reported that the differential expression of EV contents in nucleic acids (i.e., DNA, mRNA, and noncoding RNAs), proteins, and lipids may create favorable conditions for CaP invasion and metastasis ${ }^{[18-22]}$. We have recently summarized the emerging role of EVs in liquid biopsy for monitoring CaP invasion and metastasis ${ }^{[23]}$. Current high-throughput technologies for genomic, transcriptomic, and proteomic analysis are driving ground-breaking discoveries in the field of new EV biomarkers. While most of the current CaP EV studies have been focused on the mechanism of metastasis and progression and as well as between cellcell communications ${ }^{[24,25]}$, only a few reliable EV biomarkers for the risk classification of CaP were clinically applied.

In this commentary, we have to emphasize a very important milestone study, highlighting the clinical importance of EV biomarkers in $\mathrm{CaP}$ stratification, which is the discovery of a novel urine $\mathrm{EV}$ gene expression assay [the ExoDx Prostate IntelliScore (EPI) urine exosome assay] to differentiate high-grade $\mathrm{CaP}$ from low-grade $\mathrm{CaP}$ and avoid unnecessary biopsies ${ }^{[26]}$. This test has been approved by the United States Food and Drug Administration with the "Breakthrough Device" designation. The EPI test is a urine EV gene expression assay that does not require pre-collection digital rectal exam and relies on the isolation and analysis of urinary EVs. In the EPI test, first catch urine samples $(25-50 \mathrm{~mL})$ are collected and EVs are isolated by a proprietary ultrafiltration centrifugation technique. After extracting exosomal RNAs, the RNA copy numbers from three genes (i.e., ERG, PCA3, and SPDEF) are determined by RT-qPCR. EPI is a noninvasive, easy-to-use, urine EV-RNA assay that has been validated across three independent prospective multicenter clinical trials with 1212 subjects $^{[27]}$. The test can discriminate high-grade ( $\geq$ GG2) from lowgrade (GG1) cancer and benign disease. EPI effectively guides the biopsy-decision process independent of PSA and other standard-of-care factors. The absence of clinical variables in the EPI algorithm represents an important differentiator from other assays predicting high-grade $\mathrm{CaP}$, including $4 \mathrm{~K}$ score test (OPKO Diagnostics, Miami, FL) and SelectMDX (MDx Health, Irvine, CA). As EPI performance is based on gene expression only, this assay is more accurate than existing risk assessment methods such as clinical features. There is an option for the urologist to introduce other parameters, such as obesity status, underlying genetics, and race, for developing a more personalized risk assignment at both initial and or repeat biopsy time-points. Among urologists, 68\% reported that the EPI test influenced their biopsy decision with respect to selecting the right patients to biopsy at the right time, thereby improving their ability to identify clinically significant disease and reduce biopsies when the test was negative ${ }^{[28]}$. Although the EPI test has achieved some satisfactory results, future studies need to incorporate it in determining the use of MRI and inclusion of the EPI-risk score into an algorithm that includes the PIRADS designation and other clinical variables. More clinical trials still need to further confirm its clinical value. In addition to the EPI test, early diagnosis or efficient prognostic EV biomarkers are warranted for improving risk stratification, personalized postoperative adjuvant therapy, and prognostication of $\mathrm{CaP}$ patients for clinical translation in the future.

Although the analysis of EV contents is promising in the early diagnosis and progression grading of CaP, the application of the EV-based liquid biopsy in the clinic is still facing challenges. Due to the heterogenous nature of human samples and complications in isolation, it is ideal to optimize the isolation technique to obtain relatively homogeneous EV cargoes with reproducible, high-yielding and throughput, and scale-up capability. The contaminations in blood (e.g., high-abundance blood proteins and apolipoproteins) and urine (e.g., mucoprotein, also called as the Tamm-Horsfall protein) need to be removed before genomic and proteomic analysis.

EVs act as cellular messengers and have been shown to transfer proteins and nucleic acids between tumor cells that influence tumor initiation, proliferation, progression, and metastasis ${ }^{[29-31]}$. An increasing number of studies have screened candidate biomarkers from body fluids that may be used to diagnose CaP. Due to the 


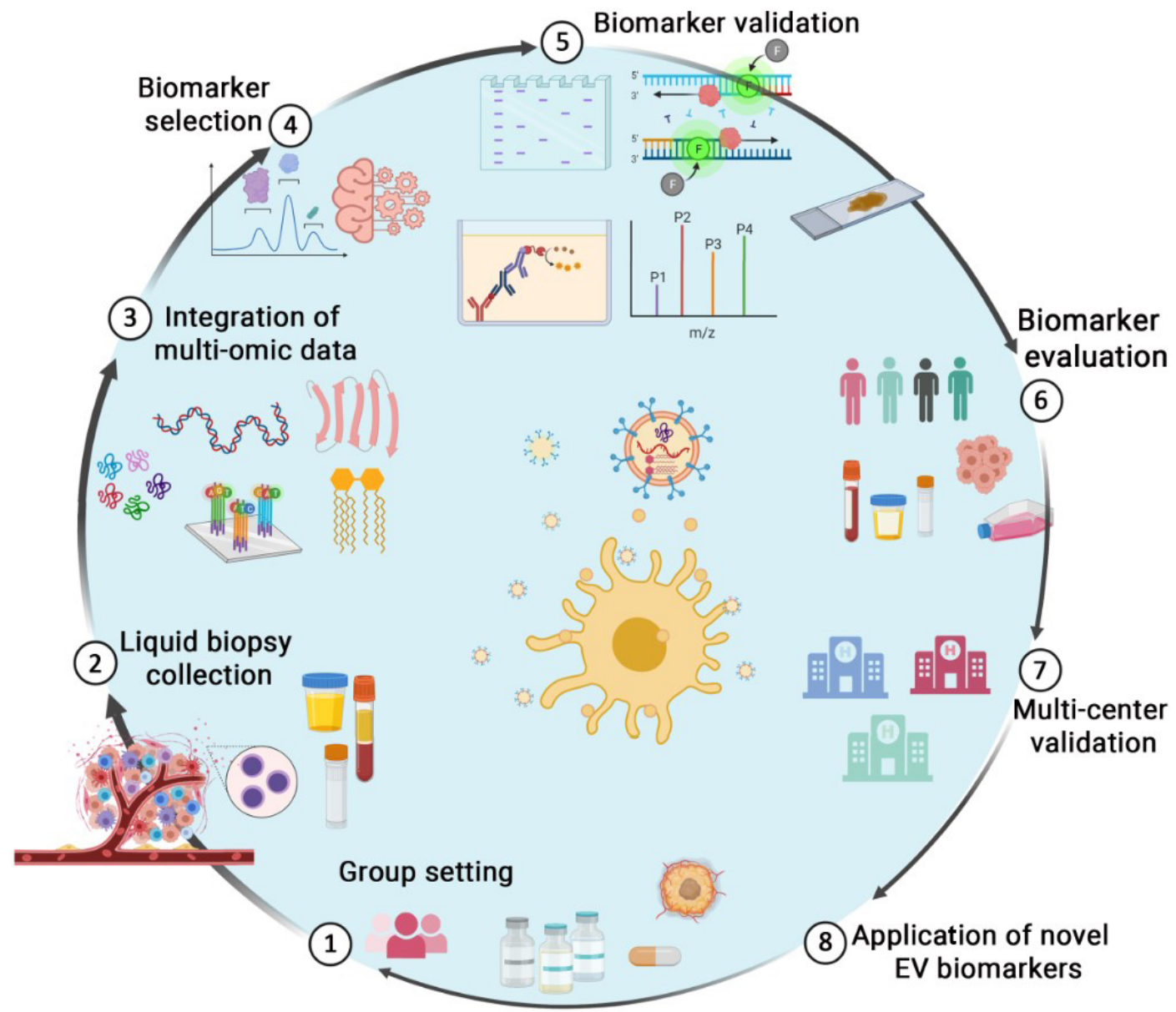

Figure 1. Overview of EV biomarker discovery and validation for CaP personalized treatment decision. Step 1. Group different stages of $\mathrm{CaP}$ patients and healthy control subjects. Step 2. Collect human samples including blood, urine, and semen. Step 3. Integrate multiomic technology including genomics, transcriptomics, proteomics and lipidomics for profiling. Step 4. Select candidate biomarkers by machine learning and establish machine learning models. Step 5. Validate selected biomarkers by different methods such as WB, qRTPCR, ELISA, PRM, and immunofluorescence in samples such as blood, urine, tissue, and cultured cell lines. Step 6. Evaluate selected biomarkers in a large set of independent patient samples. Step 7. Multi-center EV biomarker diagnosis performance verification. Step 8. Novel EV biomarkers for early diagnosis and patient grading, and for precision medicine. ELISA: Enzyme-linked immunosorbent assay; CaP: prostate cancer; EV: extracellular vesicle; qRT-PCR: quantitative reverse transcription PCR; PRM: parallel reaction monitoring; WB: western blot.

heterogeneity and variety of genetic backgrounds of patients, the lack of data from large-scale samples for the validation of $\mathrm{CaP}$ biomarkers is the main deterrent to translating the potential EV biomarkers from bench to the bedside. In order to discover specific and sensitive biomarkers of tumors in diagnosis and screening, more multidisciplinary technologies and collaborations are highly expected in the near future.

The advances in high-throughput and modern omics technologies such as genomics, transcriptomics and proteomics have greatly promoted EV biomarker research in the recent decade. Machine learning has been applied to integrate multi-omics sequencing data, which are still generated at ever-growing rates and scales. Machine learning can lead to a high-quality performance for liquid biopsy-based diagnosis for multiple human cancers $^{[15]}$ and holds promise for EV-based CaP liquid biopsy. Comprehensive multi-omics data analysis with machine learning has been a frontier in cancer genomics ${ }^{[32]}$ and should be performed in $\mathrm{CaP}$ EV biomarker research in the future [Figure 1]. 
Oncogenesis is driven by a complex and intricately controlled gene expression programming related to molecular-level variation in many genes. Due to the high heterogeneity and intricacy, a single biomarker does not fully characterize tumor properties, and more accurate predictions using multi-parameter markers are required ${ }^{[3,343}$. A set of candidate biomarkers can be evaluated for their differences in abundance between patients and normal controls, resulting in more robust predictive and diagnostic capacities ${ }^{[35]}$. For CaP EVbased biomarker research, a comprehensive pipeline for discovery and validation is shown in Figure 1 .

Most importantly, these putative EV biomarkers need further validation independently at multiple levels, such as blood, urine, tumor tissue, and cell lines, to increase their specificity and sensitivity before clinical application. During this stage, the sensitivity and specificity of the selected EV markers need to be compared with the current PSA test, MRI imaging, and tissue biopsy. These markers need to ensure they are diseasespecific, rather than trial-dependent. Assessing multi-center EV biomarker diagnosis performance in CaP is necessary to further evaluate the clinical value of these biomarker set prior to its widespread use. These EV biomarkers can be used to diagnose $\mathrm{CaP}$ and predict the stages of cancer and the tumor biological activity. This EV-based liquid biopsy can guide clinicians in choosing the best treatment methods for an individual $\mathrm{CaP}$ patient and significantly improve their prognosis.

\section{DECLARATIONS}

\section{Authors' contributions}

Made substantial contributions to conception, design, writing, and editing of the paper, and performed literature searches and interpretation: Hand M, Pang B, Zhou C, Li X, Wang Q, Jiang J, Li Y

\section{Availability of data and materials}

Not applicable.

\section{Financial support and sponsorship}

This work was supported in part by the Basic Public Welfare Research Project of Zhejiang Province (LGF22H050001-Han M), Medical Health Science and Technology Project of Zhejiang Provincial Health Commission (2020ZH007-Jiang JH and 2019KY575-Zhou C), Ningbo Science Foundation (202002N3193Jiang JH). The St. George Hospital Cancer Research Trust Fund. Pang B is supported by the University International Postgraduate Award (UIPA) Scholarship from UNSW Sydney, Australia.

\section{Conflicts of interest}

All authors declared that there are no conflicts of interest.

\section{Ethical approval and consent to participate}

Not applicable.

\section{Consent for publication}

Not applicable.

\section{Copyright}

(c) The Author(s) 2022.

\section{REFERENCES}

1. Liu Z, Jiang Y, Fang Q, et al. Future of cancer incidence in Shanghai, China: predicting the burden upon the ageing population. Cancer Epidemiol 2019;60:8-15. DOI PubMed

2. Shin S, Saito E, Sawada N, et al; JPHC Study Group. Dietary patterns and prostate cancer risk in Japanese: the Japan Public Health Center-based Prospective Study (JPHC Study). Cancer Causes Control 2018;29:589-600. DOI PubMed

3. Cao W, Chen HD, Yu YW, Li N, Chen WQ. Changing profiles of cancer burden worldwide and in China: a secondary analysis of the 
global cancer statistics 2020. Chin Med J (Engl) 2021;134:783-91. DOI PubMed PMC

4. Zhu Y, Mo M, Wei Y, et al. Epidemiology and genomics of prostate cancer in Asian men. Nat Rev Urol 2021;18:282-301. DOI PubMed

5. Siegel DA, O'Neil ME, Richards TB, Dowling NF, Weir HK. Prostate cancer incidence and survival, by stage and race/ethnicity United States, 2001-2017. MMWR Morb Mortal Wkly Rep 2020;69:1473-80. DOI PubMed PMC

6. Howard N, Clementino M, Kim D, et al. New developments in mechanisms of prostate cancer progression. Semin Cancer Biol 2019;57:111-6. DOI PubMed

7. Prensner JR, Rubin MA, Wei JT, Chinnaiyan AM. Beyond PSA: the next generation of prostate cancer biomarkers. Sci Transl Med 2012;4:127rv3. DOI PubMed PMC

8. Thompson IM, Pauler DK, Goodman PJ, et al. Prevalence of prostate cancer among men with a prostate-specific antigen level < or $=4.0$ ng per milliliter. $N$ Engl J Med 2004;350:2239-46. DOI PubMed

9. Kohaar I, Petrovics G, Srivastava S. A rich array of prostate cancer molecular biomarkers: opportunities and challenges. Int J Mol Sci 2019;20:1813. DOI PubMed PMC

10. Thompson JE, van Leeuwen PJ, Moses D, et al. The diagnostic performance of multiparametric magnetic resonance imaging to detect significant prostate cancer. J Urol 2016;195:1428-35. DOI PubMed

11. Marrugo-Ramírez J, Mir M, Samitier J. Blood-based cancer biomarkers in liquid biopsy: a promising non-invasive alternative to tissue biopsy. Int J Mol Sci 2018;19:2877. DOI PubMed PMC

12. De Rubis G, Rajeev Krishnan S, Bebawy M. Liquid biopsies in cancer diagnosis, monitoring, and prognosis. Trends Pharmacol Sci 2019;40:172-86. DOI PubMed

13. Fitts CA, Ji N, Li Y, Tan C. Exploiting exosomes in cancer liquid biopsies and drug delivery. Adv Healthc Mater 2019;8:e1801268. DOI PubMed

14. Vergauwen G, Tulkens J, Pinheiro C, et al. Robust sequential biophysical fractionation of blood plasma to study variations in the biomolecular landscape of systemically circulating extracellular vesicles across clinical conditions. J Extracell Vesicles 2021;10:e12122. DOI PubMed PMC

15. Hoshino A, Kim HS, Bojmar L, et al. Extracellular vesicle and particle biomarkers define multiple human cancers. Cell 2020;182:1044-61.e18. DOI PubMed PMC

16. Kalluri R. The biology and function of exosomes in cancer. J Clin Invest 2016;126:1208-15. DOI PubMed PMC

17. Pang B, Zhu Y, Ni J, et al. Extracellular vesicles: the next generation of biomarkers for liquid biopsy-based prostate cancer diagnosis. Theranostics 2020;10:2309-26. DOI PubMed PMC

18. Zhang Y, Zhao J, Ding M, et al. Loss of exosomal miR-146a-5p from cancer-associated fibroblasts after androgen deprivation therapy contributes to prostate cancer metastasis. J Exp Clin Cancer Res 2020;39:282. DOI PubMed PMC

19. Zhang Y, Chen B, Xu N, et al. Exosomes promote the transition of androgen-dependent prostate cancer cells into androgenindependent manner through up-regulating the heme oxygenase-1. Int J Nanomedicine 2021;16:315-27. DOI PubMed PMC

20. Mo C, Huang B, Zhuang J, Jiang S, Guo S, Mao X. LncRNA nuclear-enriched abundant transcript 1 shuttled by prostate cancer cellssecreted exosomes initiates osteoblastic phenotypes in the bone metastatic microenvironment via miR-205-5p/runt-related transcription factor 2/splicing factor proline- and glutamine-rich/polypyrimidine tract-binding protein 2 axis. Clin Transl Med 2021;11:e493. DOI PubMed PMC

21. Zavridou M, Strati A, Bournakis E, Smilkou S, Tserpeli V, Lianidou E. Prognostic significance of gene expression and DNA methylation markers in circulating tumor cells and paired plasma derived exosomes in metastatic castration resistant prostate cancer. Cancers (Basel) 2021;13:780. DOI PubMed PMC

22. Yi X, Li Y, Hu X, Wang F, Liu T. Changes in phospholipid metabolism in exosomes of hormone-sensitive and hormone-resistant prostate cancer cells. $J$ Cancer 2021;12:2893-902. DOI PubMed PMC

23. Gao Z, Pang B, Li J, Gao N, Fan T, Li Y. Emerging role of exosomes in liquid biopsy for monitoring prostate cancer invasion and metastasis. Front Cell Dev Biol 2021;9:679527. DOI PubMed PMC

24. Peinado H, Zhang H, Matei IR, et al. Pre-metastatic niches: organ-specific homes for metastases. Nat Rev Cancer 2017;17:302-17. DOI PubMed

25. Henrich SE, McMahon KM, Plebanek MP, et al. Prostate cancer extracellular vesicles mediate intercellular communication with bone marrow cells and promote metastasis in a cholesterol-dependent manner. J Extracell Vesicles 2020;10:e12042. DOI PubMed PMC

26. McKiernan J, Donovan MJ, O'Neill V, et al. A novel urine exosome gene expression assay to predict high-grade prostate cancer at initial biopsy. JAMA Oncol 2016;2:882-9. DOI PubMed

27. Margolis E, Brown G, Partin A, et al. Predicting high-grade prostate cancer at initial biopsy: clinical performance of the ExoDx (EPI) prostate intelliscore test in three independent prospective studies. Prostate Cancer Prostatic Dis 2021. DOI PubMed

28. Tutrone R, Donovan MJ, Torkler P, et al. Clinical utility of the exosome based ExoDx Prostate(IntelliScore) EPI test in men presenting for initial Biopsy with a PSA 2-10 ng/mL. Prostate Cancer Prostatic Dis 2020;23:607-14. DOI PubMed PMC

29. Zhang L, Zhang S, Yao J, et al. Microenvironment-induced PTEN loss by exosomal microRNA primes brain metastasis outgrowth. Nature 2015;527:100-4. DOI PubMed PMC

30. Luga V, Zhang L, Viloria-Petit AM, et al. Exosomes mediate stromal mobilization of autocrine Wnt-PCP signaling in breast cancer cell migration. Cell 2012;151:1542-56. DOI PubMed

31. Datta A, Kim H, Lal M, et al. Manumycin A suppresses exosome biogenesis and secretion via targeted inhibition of Ras/Raf/ERK1/2 signaling and hnRNP H1 in castration-resistant prostate cancer cells. Cancer Lett 2017;408:73-81. DOI PubMed PMC

32. Ko J, Bhagwat N, Yee SS, et al. Combining machine learning and nanofluidic technology to diagnose pancreatic cancer using 
exosomes. ACS Nano 2017;11:11182-93. DOI PubMed

33. El-Khoury V, Schritz A, Kim SY, et al. Identification of a blood-based protein biomarker panel for lung cancer detection. Cancers (Basel) 2020;12:1629. DOI PubMed PMC

34. Zhang YH, Jin M, Li J, Kong X. Identifying circulating miRNA biomarkers for early diagnosis and monitoring of lung cancer. Biochim Biophys Acta Mol Basis Dis 2020;1866:165847. DOI PubMed

35. Xu M, Deng J, Xu K, et al. In-depth serum proteomics reveals biomarkers of psoriasis severity and response to traditional Chinese medicine. Theranostics 2019;9:2475-88. DOI PubMed PMC 